\title{
VERIFICATION OF RADIAL DISPLACEMENT CORRECTION EFFECT IN INSTRUMENTED INDENTATION TESTING
}

\author{
Y. Tanaka ${ }^{1}$, Y. Seino ${ }^{2}$, K. Hattori ${ }^{3}$ \\ ${ }^{1}$ National Metrology Institute of Japan, National Institute of Advanced Industrial Science and Technology, \\ Tsukuba, Japan, yukimi.tanaka@aist.go.jp \\ ${ }^{2}$ National Metrology Institute of Japan, National Institute of Advanced Industrial Science and Technology, \\ Tsukuba, Japan, y.seino@aist.go.jp \\ ${ }^{3}$ National Metrology Institute of Japan, National Institute of Advanced Industrial Science and Technology, \\ Tsukuba, Japan, hattori-k@aist.go.jp
}

\begin{abstract}
:
The radial displacement had been ignored in the analysis of unloading curve of instrumented indentation test. There are two representative methods of radial displacement correction, proposed by Hay et al. [J. Mater. Res., 14, 2296, (1999)] and Chudoba-Jennett [J. Phys. D, 41, 215407, (2008)]. In this study, we examine the effect of radial displacement corrections in finite element analysis and experiments.
\end{abstract}

Keywords: Instrumented indentation testing, radial displacement

\section{INTRODUCTION}

Instrumented indentation testing is a useful method to evaluate local mechanical properties such as the elastic modulus $(E)$ and hardness $(H)$. Sneddon's solution indicates the relationship between load $(P)$ and displacement $(h)$ in elastic half space [1]. After that, Oliver and Pharr showed the calculation elastic modulus and hardness in elasticplastic deformation, by following equations [2]:

$E_{r}=S /(2 a)$

$H=P_{m} /\left(\pi a^{2}\right)$

where $P_{\mathrm{m}}$ is the maximum load, $S$ is the unloading stiffness at the maximum depth, and $a$ is the contact radius. $E_{\mathrm{r}}$ is reduced modulus. However, it is pointed out that this method can cause errors in measuring elastic modulus and hardness due to ignoring radial displacement $\left(u_{\mathrm{r}}\right)$ [3-4]. The correction factor of radial displacement is basically shown as $\gamma=1+\left|u_{\mathrm{r}} / a\right|$. There are two representative method of radial displacement correction, proposed by Hay et al. [3] and Chudoba-Jennett [4].

Hay et al. [3] pointed out that indentation by a rigid cone requires larger loads than those predicted by Sneddon's analysis due to the radial surface displacements. They proposed that the following correction:

$$
\begin{aligned}
& E_{r_{-} \text {corr }}=\left(1 / \gamma_{H}\right) \cdot S /(2 a) \\
& \gamma_{H}=1+\frac{1-2 v}{4(1-v)} \cot \theta \\
& \gamma_{H}=\pi \frac{\frac{\pi}{4}+0.1548 \cot \theta \frac{1-2 v}{4(1-v)}}{\left[\frac{\pi}{2}-0.8312 \cot \theta \frac{1-2 v}{4(1-v)}\right]^{2}}
\end{aligned}
$$

where $a=\left(2 h_{\mathrm{m}} \tan \theta\right) / \pi, \theta$ is the indenter angle and $v$ is the Poisson's ratio. Eq.(4) is suitable for cubecorners, and Eq.(5) is for indenters whose equivalent cone angles are $70.3^{\circ}$.

Chudoba and Jennett [4] assumed elastic-plastic deformation and proposed the following correction factor based on the radial displacement at the contact edge:

$\gamma_{C}=1+[(1-2 v)(1+v) H] /(2 E) \cdot \cos \alpha_{r}$

where $\alpha_{\mathrm{r}}$ assumes the residual angle between the face of the impression and the original surface plane. ISO14577 recommends the correction by ChudobaJennett method [5]. In the ISO, correcting indentation results for radial displacement increases the elastic modulus as given in following equation:

$$
E_{r_{-} \text {corr }}=\gamma_{C} \cdot S /(2 a)
$$

where $a$ is determined by real (unloaded) indenter shape. The contact radius satisfying $S=2 E_{\mathrm{r}} a$ is the one during loading and smaller than after unloading. Note that $\left(90^{\circ}-\theta\right)$ is used instead of $\alpha_{\mathrm{r}}$ when $\gamma_{\mathrm{C}}$ is calculated in the ISO, because it is difficult to obtain the residual angle from load-displacement data.

The correction factors $\gamma_{\mathrm{H}}$ and $\gamma_{\mathrm{C}}$ are both $\gamma>1$. Hay's correction decrease $E_{\mathrm{r}}$ as shown in Eq.(3), whereas Chudoba's correction increase $E_{\mathrm{r}}$ as shown in Eq.(7). These methods are inconsistent in the direction of correcting elastic modulus, although 
they both assume that the surface point is displaced inward under pressure.

In actual experiment, the area function, $A_{c}$, is defined by experimental data of the reference materials whose $E$ is known, by following equation:

$$
A_{c}=(\pi / 4) \cdot\left(S / E_{r}\right)^{2}
$$

Fused silica (FS) is often used as reference material. Hay et al. mentioned that Eq.(8) led to an overestimation of $A_{c}$ by $20 \%$ when FS was used. On the other hand, Chudoba and Jennett indicated that $A_{\mathrm{c}}$ determined by FS was underestimated by $13 \%$ compared with the area function from direct measurement by AFM. Correction ways of area function are also different between Hay's and Chudoba's methods.

In this study, the effectiveness of these methods is verified by actual experiments and simulation of finite element analysis (FEA). In experiments, the materials whose elastic moduli are known are measured by indentation testing. The effects of radial displacement corrections are investigated by comparing to theoretical elastic modulus. In FEA simulation, the contact radius with radial displacement is considered in fully elastic deformation and elastic-plastic deformation. Then, we discuss the accurate correction method which can be applied to actual measurement.

\section{METHODS}

\subsection{Experiments}

To verify the effects of the correction methods, nanoindentation-tests were carried out by using iMicro (Nanomechanics) with a Berkovich indenter. To calculate the elastic modulus, continuous stiffness measurements were used. Measured materials were sapphire $\left(\mathrm{Al}_{2} \mathrm{O}_{3}\right)$, tungsten $(\mathrm{W})$, Aluminium (Al), SiC, GaN, MgO, Molybdenum (Mo), and $\mathrm{CaF}_{2}$. These materials have single crystal structure. Area function, $A_{\mathrm{c}}\left(h_{\mathrm{c}}\right)$, was determined by measuring data of FS $(E=72 \mathrm{GPa}, v=0.17)$ or BK7 glass $(E=83 \mathrm{GPa}, v=0.21)$ as reference materials.
It is necessary to consider both radial displacement effects of reference and measured materials. The significant difference due to the radial displacement was found for FS [3-4]. FS and BK7 were used as reference materials to consider the difference between reference materials.

\subsection{Finite element analysis (FEA)}

To confirm the validity of the correction methods, we simulated the fully elastic half-space and the elastic-plastic half-space by FEA using COMSOL Multiphysics.

Fully elastic deformation with a $70.3^{\circ}$ rigid conical indenter was simulated with a twodimensional axisymmetric model. The examined materials have different Poisson's ratios $(v=0.0$, $0.1,0.2,0.3,0.4$ and 0.5 ). The elastic modulus was fixed with $100 \mathrm{GPa}$. In addition, elastic-plastic deformation with a $70.3^{\circ}$ rigid conical indenter was simulated with a two-dimensional axisymmetric model. The elastic-plastic materials were modelled by Swift's law. The examined materials have different work-hardening coefficients $(n=0.0,0.2$, and 0.4), Poisson's ratios ( $v=0.1,0.2,0.3$ and 0.4$)$, and ratios between elastic modulus and yield stress $\left(E / \sigma_{\mathrm{y}}=10,25,50\right.$, and 100$)$.

\section{RESULTS}

\subsection{Experiments}

Table 1 shows the elastic moduli with and without radial displacement correction by Hay and Chudoba methods in the experiments. Corrected elastic moduli of measured materials depend on the radial displacement of both themselves and the reference material, because $A_{\mathrm{c}}\left(h_{\mathrm{c}}\right)$ is corrected by the radial displacement of the reference material before applying the correction method to measured materials. In the case of using $A_{\mathrm{c}}$ function from FS, most of corrected elastic moduli by Chudoba method got closer to the theoretical values than that by Hay method. However, in the $A_{\mathrm{c}}$ function from BK7, elastic moduli of Hay method approach the

Table 1: Elastic moduli obtained from experiments.

\begin{tabular}{|c|c|c|c|c|c|c|c|c|}
\hline \multirow{3}{*}{ Samples } & \multirow{3}{*}{$v$} & \multirow{3}{*}{$\begin{array}{c}\text { Theo. } \\
\text { E }\end{array}$} & \multicolumn{6}{|c|}{ Experimental $E$, GPa } \\
\hline & & & \multicolumn{3}{|c|}{$A_{\mathrm{c}}\left(h_{\mathrm{c}}\right)$ from $\mathrm{FS}$} & \multicolumn{3}{|c|}{$A_{\mathrm{c}}\left(h_{\mathrm{c}}\right)$ from $\mathrm{BK} 7$} \\
\hline & & & No-corr. & Hay & Chudoba & No-corr. & Hay & Chudoba \\
\hline $\mathrm{SiC}$ & 0.07 & 475 & $576 \pm 17$ & $567 \pm 17$ & $568 \pm 18$ & $554 \pm 27$ & $542 \pm 26$ & $554 \pm 28$ \\
\hline $\mathrm{Al}_{2} \mathrm{O}_{3}$ & 0.16 & 422 & $483 \pm 9$ & $482 \pm 9$ & $473 \pm 9$ & $465 \pm 13$ & $461 \pm 13$ & $461 \pm 14$ \\
\hline $\mathrm{GaN}$ & 0.18 & 320 & $326 \pm 3$ & $326 \pm 3$ & $318 \pm 3$ & $314 \pm 6$ & $313 \pm 6$ & $311 \pm 6$ \\
\hline $\mathrm{CaF}_{2}$ & 0.21 & 120 & $132 \pm 3$ & $133 \pm 3$ & $127 \pm 3$ & $127 \pm 3$ & $127 \pm 3$ & $124 \pm 3$ \\
\hline $\mathrm{MgO}$ & 0.24 & 288 & $315 \pm 5$ & $319 \pm 5$ & $305 \pm 5$ & $304 \pm 6$ & $306 \pm 7$ & $298 \pm 7$ \\
\hline Mo & 0.28 & 332 & $338 \pm 7$ & $345 \pm 7$ & $324 \pm 6$ & $325 \pm 7$ & $329 \pm 7$ & $316 \pm 7$ \\
\hline W & 0.28 & 409 & $411 \pm 10$ & $419 \pm 10$ & $394 \pm 10$ & $394 \pm 10$ & $399 \pm 10$ & $383 \pm 10$ \\
\hline $\mathrm{Al}$ & 0.36 & 68 & $70 \pm 3$ & $73 \pm 3$ & $67 \pm 3$ & $68 \pm 3$ & $70 \pm 3$ & $66 \pm 3$ \\
\hline \multicolumn{3}{|c|}{$\begin{array}{c}\text { Average absolute } \\
\text { difference from theo. }\end{array}$} & $7.8 \%$ & $8.8 \%$ & $6.4 \%$ & $5.7 \%$ & $5.5 \%$ & $6.2 \%$ \\
\hline
\end{tabular}

Values reported as mean \pm standard deviation. 
theoretical values than that of Chudoba method. It is unclear that the effectiveness of Hay and Chudoba method only from experiments, thus, we conducted the simulation of FEA.

\subsection{Fully elastic deformation by FEA}

For verifying the way of correcting radial displacements, the indentation was simulated by FEA. Figure 1 shows the FEA simulation results of fully elastic deformation. There are three ways to calculate contact radius, $a_{\mathrm{SN}}$ based on Sneddon's solution $a=\left(2 h_{\mathrm{m}} \tan \theta\right) / \pi, a_{\mathrm{FEA}}$ obtained from FEA results, and $a_{\mathrm{E}}$ calculated from $a=S /\left(2 E_{\mathrm{r}}\right)$. In $v=0.5$, $a_{\mathrm{SN}}, a_{\mathrm{FEA}}$ and $a_{\mathrm{E}}$ were consistent each other because of $u_{\mathrm{r}}=0$. However, they were not consistent in $v=$ 0 . The surface point is displaced inward, thus $a_{\mathrm{FEA}}$ is smaller than $a_{\mathrm{SN}}$. In contrast, $a_{\mathrm{E}}$ is larger than $a_{\mathrm{SN}}$, because the indentation load and stiffness of FEA is higher than that of Sneddon's solution. As shown in figure $1 \mathrm{~b}, a_{\mathrm{E}}$ and $a_{\mathrm{FEA}}$ depend on Poisson's ratio but their tendencies are different. In addition, the ratio of the correction by Hay and Chudoba methods is also shown in figure $1 \mathrm{~b}$. Note that $P_{\mathrm{m}} /\left(\pi a^{2}\right)$ was used instead of $H$ in Chudoba's factor because the fully elastic half space was assumed. These results suggest that Hay's method can be useful for $a_{\mathrm{E}}$ estimation, and Chudoba's method can estimate $a_{\text {FEA. }}$.

\subsection{Elastic-plastic deformation by FEA}

For verifying the way of correcting radial displacements in experiments, the elastic-plastic indentation was simulated by FEA. Note that Hay's method doesn't assume elastic-plastic deformation. Figure 2 shows the FEA simulation results of elastic-plastic deformation. Figure $2 \mathrm{a}$ shows the relationship between the amount of radial displacement and $h_{\mathrm{f}} / h_{\mathrm{m}}$, where $h_{\mathrm{m}}$ is the maximum depth and $h_{\mathrm{f}}$ is the final depth. $u_{\mathrm{r}} / a$ are directly obtained from FEA, $u_{\mathrm{r}}$ indicates the difference between loading and unloading at $r=a$. FEA results indicate that the radial displacement depends on the Poisson's ratio and the amount of elastic deformation. $\left|u_{\mathrm{r}} / a\right|$ values calculated by ChudobaJennett method agreed with FEA results, whereas the estimated values by Hay et al. method which depends on the Poisson's ratio were different from FEA values. This result suggest that ChudobaJennett method is suitable for estimating $\left|u_{\mathrm{r}} / a\right|$ in the actual experiments.

The Figures $2 b$ and $2 c$ indicate the difference in contact radii calculated by three ways, $a_{\mathrm{E}}, a_{\mathrm{FEA}}$ and $a_{\mathrm{OP}}$ which is obtained by $h_{\mathrm{c}}=h_{\mathrm{m}}-\varepsilon\left(P_{\mathrm{m}} / S\right)$. When sink-in behaviour is easy to be occurred such as $n=$ 0.4 , the FEA results were similar to those of fully elastic deformation. $a_{\mathrm{E}} / a_{\mathrm{OP}}$ is consistent with the correction factor of Hay's method whereas $a_{\mathrm{FEA}} / a_{\mathrm{OP}}$ is consistent with that of Chudoba's method. However, when pile-up behavior is easy to be

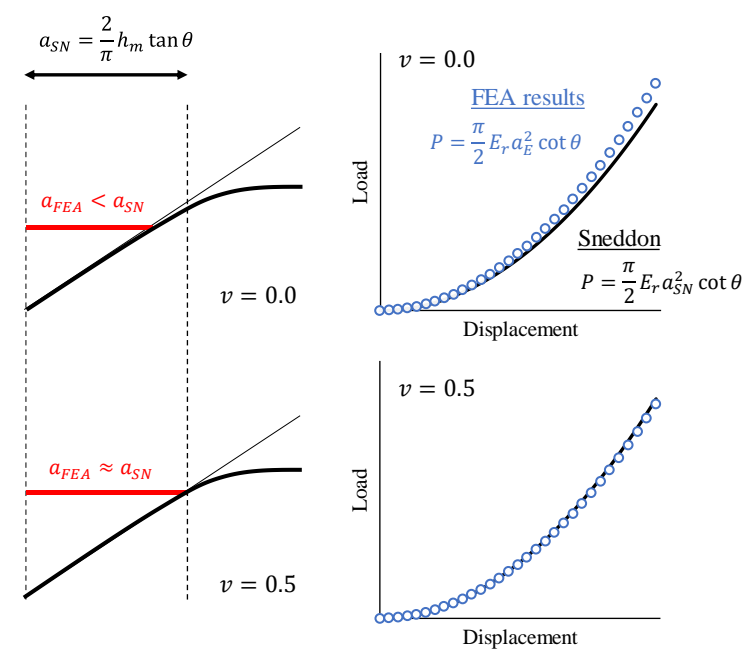

(a) Scheme of FEA simulation at $v=0.0$ and $v=0.5$

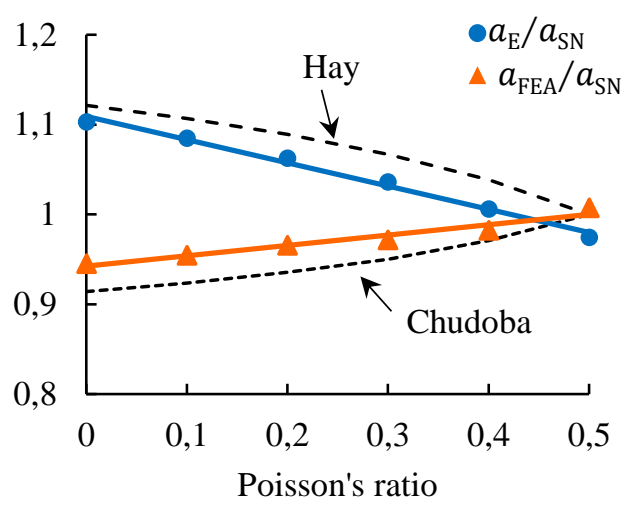

(b) Difference in calculated contact radius

Figure 2: FEA simulation results of fully elastic deformation considering radial displacement effects. $a_{\mathrm{SN}}$ : contact radius based on Sneddon's solution. $a_{\mathrm{FEA}}$ : contact radius obtained from FEA. $a_{\mathrm{E}}$ : contact radius calculated by $a_{E}=S /\left(2 E_{r}\right)$.

occurred such as $n=0.0$, the variation of $a_{\mathrm{E}} / a_{\mathrm{OP}}$ and $a_{\mathrm{FEA}} / a_{\mathrm{OP}}$ was large, because the estimation of $h_{\mathrm{c}}$ and $a_{\mathrm{OP}}$ might be wrong.

\section{DISCUSSION}

In fully elastic deformation, our results of FEA simulation suggest $a_{\mathrm{FEA}}<a_{\mathrm{SN}}<a_{\mathrm{E}}$. Schwarzer indicated the contact radius considering radial displacement was smaller than that based on Sneddon's analysis, by using basic principles of linear elasticity [6]. In addition, Hay et al. showed the load from FEA simulation was larger than that from Sneddon's analysis, and the effective cone angle was larger than the original angle [3]. These previous studies are consistent with our results of FEA. It is necessary to be careful not to confuse $a$ in FEA and $a=S /\left(2 E_{\mathrm{r}}\right)$ which is used for calculating elastic modulus by Eq. (1).

The present results of FEA suggest that Hay's method can obtain the contact area for calculating 

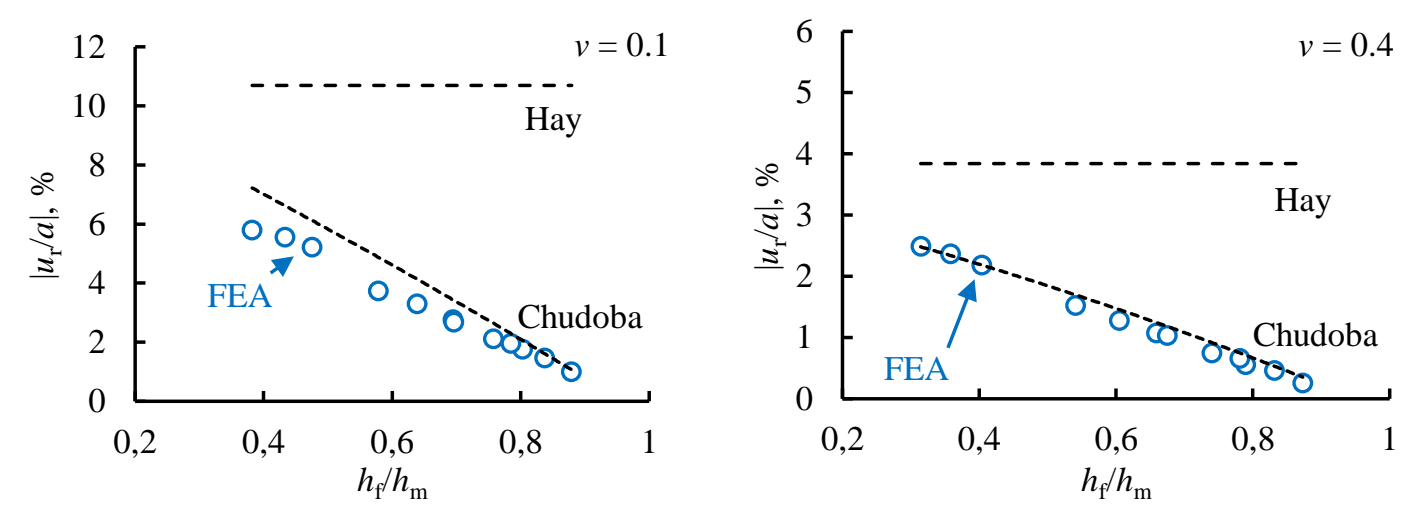

(a) The relationship between $\left|u_{\mathrm{r}} / a\right|$ and $h_{\mathrm{f}} / h_{\mathrm{m}}$ with different $E / \sigma_{\mathrm{y}}$ and $n$
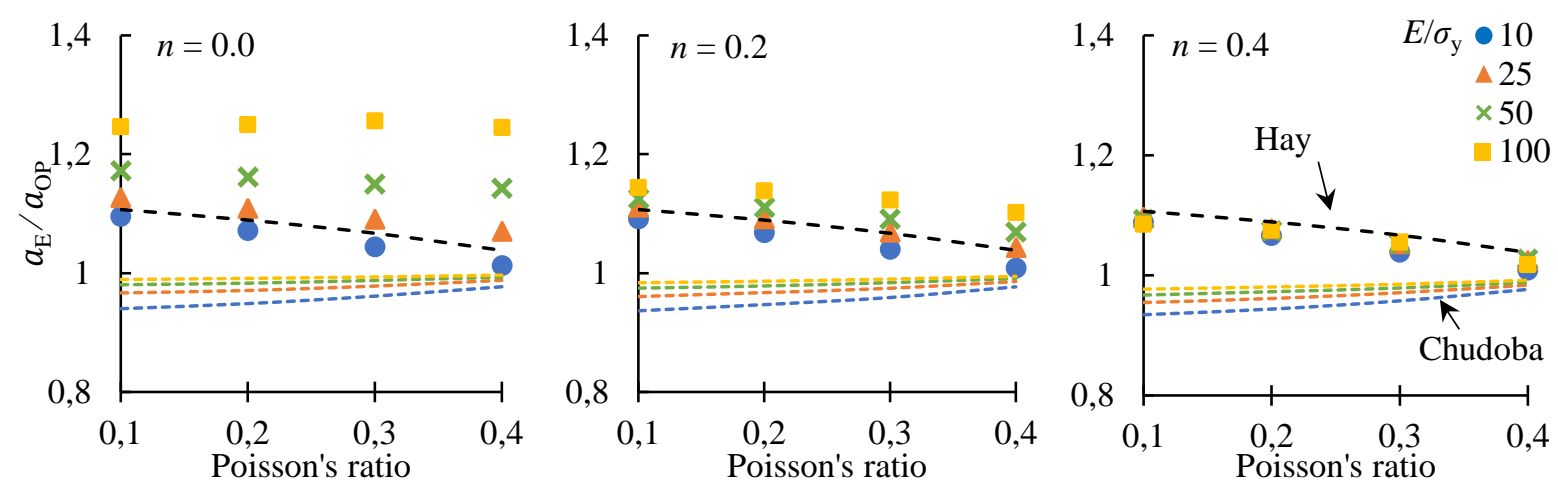

(b) Difference in $a_{\mathrm{E}} / a_{\mathrm{OP}}$ with Poisson's ratio, work-hardening coefficients and $E / \sigma_{\mathrm{y}}$ ratio. $a_{\mathrm{OP}}$ : contact radius based on Oliver-Pharr method. $a_{\mathrm{E}}$ : contact radius calculated by $a_{E}=S /\left(2 E_{r}\right)$.
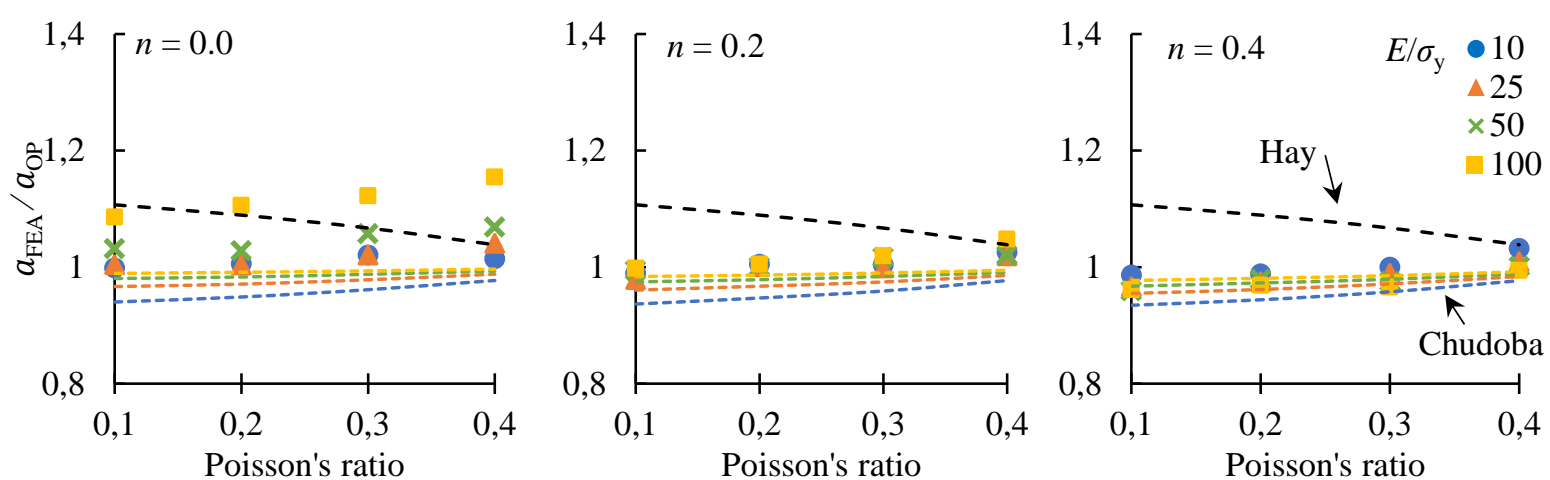

(c) Difference in $a_{\mathrm{FEA}} / a_{\mathrm{OP}}$ with Poisson's ratio, work-hardening coefficients and $E / \sigma_{\mathrm{y}}$ ratio. $a_{\mathrm{OP}}$ : contact radius based on Oliver-Pharr method. $a_{\mathrm{FEA}}$ : contact radius obtained from FEA.

Figure 2: FEA simulation results of elastic-plastic deformation considering radial displacement effects.

elastic modulus by Eq.(1), while Chudoba's method can obtain the apparent contact area during loading. Therefore, Eq.(3) of Hay's method seems to be suitable to obtain appropriate elastic modulus than Eq.(7) of Chudoba's method. However, Hay's method considered the ideal boundary condition based on Sneddon analysis (related to $a_{\mathrm{SN}}$ ), although it should consider the actual boundary condition (related to $\left.a_{\mathrm{FEA}}\right)$. This suggests that both of Hay's and Chudoba's corrections should be taken into account. Recent research shows the improved correction factor of Hay's method which is taken the boundary condition after the surface displaced inward into consideration in fully elastic deformation [7]. Furthermore, the boundary condition in elastic-plastic deformation is more complex than that in fully elastic deformation, because the amount of radial displacement is varied with $E / \sigma_{\mathrm{y}}$ and $v$ as shown in figure $2 \mathrm{a}$. It can be disputable to consider the value of correction factor in elastic-plastic deformation.

In addition, $\mathrm{Xu}$ et al. suggested that $\gamma$-factor with Berkovich indenter is almost independent of the $E / \sigma_{\mathrm{y}}$ ratio, whereas that with conical indenter depends on the $E / \sigma_{\mathrm{y}}$ [8]. If the $\gamma$-factors of reference and measured materials are the same, and it is unnecessary to correct the radial displacement effect. The further studies require to consider about the radial displacement effect of actual indentation testing using Berkovich indenters. 
In actual experiments as shown in table 1, these limited results suggest that the radial displacement correction is small because the effect of difference in reference materials is larger than that of radial displacement. Some studies pointed out that fused silica has densification effects, and higher elastic recovery than borosilicate glasses [9-10]. Thus, the determination of area function also depends on characteristics of reference material. The cause for errors of elastic moduli measured by nanoindentation requires further investigation.

\section{SUMMARY}

In this study, we examined the effect of radial displacement corrections using FEA and experiments. Hay's method can obtain the contact area for calculating appropriate elastic modulus, whereas Chudoba's method can estimate the apparent contact area during loading. The further studies require to consider that the necessary correction method for obtaining appropriate elastic moduli and hardness values in actual indentation testing.

\section{REFERENCES}

[1] I. N. Sneddon, "The relation between load and penetration in the axisymmetric Boussinesq problem for a punch of arbitrary profile", Int. J. Engng. Sci., vol. 3, pp. 47-57, 1965.

[2] W. C. Oliver, G. M. Pharr, "Measurement of hardness and elastic modulus by instrumented indentation: Advances in understanding and refinements to methodology", J. Mater. Res., vol. 19, no. 1, pp. 3-20, 2004.

[3] J. C. Hay, A. Bolshakov, G. M. Pharr, "A critical the analysis of nanoindentation data", J. Mater. Res., vol. 14, no. 6, pp. 2296-2305, 1999.

[4] T. Chudoba, N. M. Jennett, "Higher accuracy analysis of instrumented indentation data obtained with pointed indenters", J. Phys. D: Appl. Phys., vol. 41, pp. 215407, 2008.

[5] ISO 14577-1:2015, "Instrumented indentation test for hardness and materials parameters - Part 1 test method".

[6] N. Schwarzer, "Effect of lateral displacement on the surface stress distribution for cone and sphere contact”, Phil. Mag., vol. 86, pp. 5231-5237, 2006.

[7] J. H. Lee, G. M. Pharr, Y. Gao, "Corrections to the stiffness relationship in 3-sided and conical indentation problems", Int. J. Solid. Struct., vol. 166, pp. 154-166, 2019.

[8] Z.-H. Xu, X. Li, "Effects of indenter geometry and material properties on the correction factor of Sneddon's relationship for nanoindentation of elastic and elastic-plastic materials", Acta Mater., vol. 56, pp. 1399-1405, 2008.

[9] K. R. Gadelrab, F. A. Bonilla, M. Chiesa, "Densification modelling of fused silica under nanoindentation", Journal of Non-Crystalline Solids, vol. 358, pp. 392-398, 2012.

[10] S. Kasimuthumaniyan, A. A. Reddy, N. M. A. Krishnan, N. N. Gosvami, "Understanding the role of post-indentation recovery on the hardness of glasses: case of silica, borate, and borosilicate glasses", Journal of Non-Crystalline Solids, vol. 534, pp. 119955, 2020. 